'Departamento de Ciencias Neurológicas, Facultad de Medicina, Universidad de Chile. Santiago, Chile. 2Departamento de Neurociencia, Facultad de Medicina, Universidad de Chile. Santiago, Chile.

Instituto Milenio de Neurociencia Biomédica (BNI), Facultad de Medicina, Universidad de Chile. Santiago, Chile.

${ }^{4}$ Servicio de Neurología, Hospital del Salvador. Santiago, Chile. ${ }^{5}$ Servicio de Neurorradiología, Instituto de Neurocirugía Dr. Asenjo (INCA). Santiago, Chile.

Trabajo no recibió financiamiento Los autores declaran no tener conflictos de interés.

Recibido el 14 de octubre de 2019, aceptado el 10 de junio de 2020.

Correspondencia a: Dr. José Manuel Matamala José Manuel Infante 553 Providencia, Santiago, Chile. jm_matamala@med.uchile.cl

Dr. Andrés Stuardo Avenida Salvador 364 Providencia, Santiago, Chile. astuardoster@gmail.com

\section{Atrofia monomiélica distal de extremidad superior. Caso clínico}

\author{
JOSÉ MANUEL MATAMALA ${ }^{1,2,3}$, GABRIEL CEA ${ }^{1,4}$, \\ RODRIGO SALINAS ${ }^{1,4}$, AARÓN VIDAL ${ }^{5}$, INGEBORG LÓPEZ ${ }^{5}$, \\ ROBERTO MARILEO ${ }^{5}$, ANDRÉS STUARDO ${ }^{4}$
}

\section{Monomelic amyotrophy. Report of one case}

Monomelic amyotrophy, also known as Hirayama disease, is a rare lower motor neuron syndrome due to localized lower motor neuron loss in the spinal cord at the cervical level. Clinically, monomelic amyotrophy is defined by the insidious onset of unilateral atrophy and weakness involving the hand and forearm, typically beginning in the second or third decade of life. We report 19-year-old man with a two years history of slowly progressive unilateral weakness and atrophy of his right-hand muscles. Neurological examination revealed weakness and atrophy in his intrinsic hand muscles, with sparing of the abductor pollicis brevis muscle. Also, mild atrophy of the ulnar aspect of the forearm was detected with sparing of the brachioradialis muscle. Electromyography showed active and chronic neurogenic changes affecting C8 and T1 myotomes, with mild chronic neurogenic changes on C7 myotome. Magnetic resonance imaging of his cervical spine revealed spinal cord atrophy involving $C 5$ to $C 7$ segments, associated with forward displacement of the posterior wall of the dura in cervical spine flexion. The clinical features associated with the imaging and electrophysiological findings support the diagnosis of monomelic amyotrophy.

(Rev Med Chile 2021; 149: 142-146)

Key words: Muscular Atrophy: Muscular Atrophy, Spinal; Spinal Cord Diseases.

\section{L} os síndromes de motoneurona inferior (MNI) son un grupo heterogéneo de enfermedades neuromusculares que se presentan clínicamente con atrofia muscular, debilidad, e hiporeflexia ${ }^{1}$. Esto se deben a la disfunción y/o degeneración de la motoneurona alfa, daño que puede localizarse en el asta anterior, el axón motor y/o la mielina que lo rodea. Los síndromes de MNI pueden ser clasificados en hereditarios (ej. atrofia muscular espinal, AME), esporádicos (ej. esclerosis lateral amiotrófica, ELA) e inmunomediados (ej. neuropatía motora multifocal). Caben destacar también como etiología infecciosa, la poliomielitis y el síndrome postpolio. Dependiendo de la sospecha diagnóstica la información clínica debe ser complementada con pruebas electrofisiológicas, imágenes, estudio de líquido cefalorraquídeo, test genéticos y/o marcadores séricos (ej. autoanticuerpos).

La atrofia monomiélica distal de extremidad superior, conocida como enfermedad de Hirayama, es una enfermedad infrecuente de la MNI, caracterizada por inicio focal e insidioso de debilidad y atrofia de la extremidad superior, en ausencia de déficit sensitivo, bulbar o signos piramidales ${ }^{2}$. Se presenta usualmente en pacientes jóvenes (entre los 15 a 25 años), en una proporción hombre mujer de 9:1. Los síntomas progresan por un período de uno a cinco años, luego del cual se estabilizan, lo que sugiere un curso autolimitado de la enfermedad ${ }^{3}$. Su etiología es desconocida, pero un daño hipóxico-isquémico secundario a compresión medular durante la flexión cervical es la hipótesis más plausible ${ }^{4}$. Esta enfermedad ha 
sido descrita principalmente en población asiática, existiendo escasos reportes en población caucásica, y menos aún en población mestiza sudamerica$\mathrm{na}^{5-7}$. Se presenta el caso clínico de un paciente chileno con el diagnóstico de atrofia monomiélica distal de extremidad superior.

\section{Caso clínico}

Paciente de sexo masculino de 19 años de edad, chileno, sin antecedentes familiares de importancia, que consultó al servicio de neurología del Hospital del Salvador (Santiago, Chile), por cuadro de debilidad y atrofia progresiva de la mano derecha. Su historia comenzó cerca de dos años antes, con debilidad en la abducción del dedo meñique derecho y paulatina debilidad de abducción y aducción de todos los dedos de la mano, asociado a atrofia de musculatura intrínseca de la mano. Adicionalmente, refirió leve adormecimiento de la cara medial de la mano y antebrazo derecho. No mencionó ningún desencadenante capaz de acentuar la debilidad referida, específicamente no presentaba "paresia inducida por frío". Tampoco refirió dolor cervical o de cintura escapular. El examen mental y de pares craneanos fue normal, sin evidencia de Síndrome de Horner. Al examen de las extremidades destacó debilidad y marcada atrofia de musculatura intrínseca de la mano derecha, con relativa indemnidad del trofismo y fuerza del músculo abductor pollicis brevis (APB), asociado a disminución del reflejo osteotendíneo cúbito pronador (raiz C8), con indemnidad de reflejos bicipital (raíz C5), supinador (raíz C6) y tricipital (raíz C7) (Figura 1A y 1B). Además, presentaba leve atrofia de la cara medial del antebrazo derecho, con preservación del músculo braquiorradialis (Figura 1C). No se observó fasciculaciones. Destacaba leve hipoestesia subjetiva al algodón en la cara medial de la mano y antebrazo derecho. No se observó compromiso de vías largas ni amiotrofia en otros segmentos corporales. Los siguientes síndromes se plantearon como alternativas diagnósticas: (i) plexopatía braquial baja (de tronco inferior o cordón medial) o (ii) atrofia monomiélica distal de extremidad superior. Los exámenes bioquímicos y hematológicos generales resultaron normales, y una resonancia magnética (RM) de columna cervical en posición neutra, realizada 12 meses previo a la consulta, fue informada normal. El estudio electrofisiológico reveló severa

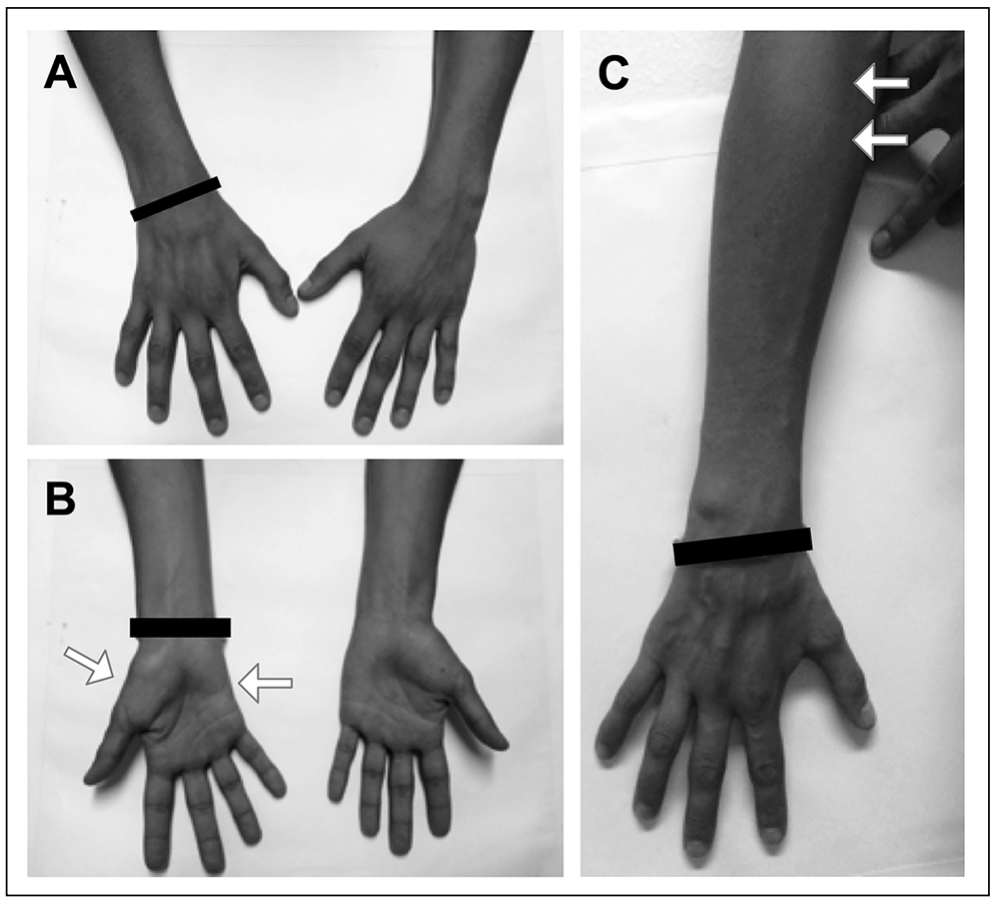

Figura 1. Hallazgos clínicos en la atrofia monomiélica distal de extremidad superior. Se observan cambios tróficos de la mano y antebrazo derecho. Se observa atrofia de la musculatura intrínsica de la mano, con relativa preservación de la musculatura tenar, en comparación con la hipotenar (A y B: flechas). Adicionalmente, es posible observar la indemidad del músculo braquiorradialis (C: flechas). 
reducción de la amplitud del potencial motor compuesto en el músculo abductor digiti minimi (ADM; 0,2 mV), con preservación de la respuesta motora en el músculo APB $(11,4 \mathrm{mV})$, asociado a velocidad de conducción motora normal en todos los nervios explorados. No se documento bloqueo de conducción. La conducción nerviosa sensitiva fue normal en los nervios mediano, cubital y antebraquial cutáneo medial a ambos lados. Ausencia de onda $\mathrm{F}$ al estimular el nervio cubital derecho, mientras en el nervio mediano derecho se registró onda $\mathrm{F}$ con latencia mínima normal. La electromiografía (EMG) mostró denervación activa y crónica en múltiples músculos correspondientes a los miotomas C8 y T1, asociada a leves signos de denervación crónica en el miotoma C7. Complementariamente, se realizaron potenciales evocados somatosensoriales estimulando el nervio cubital derecho e izquierdo (raíces C8 y T1), los cuales resultaron simétricos y con latencias en rango fisiológico. Adicionalmente, se realizó una RM de plexo braquial que resultó normal. Finalmente, se realizó una nueva RM de columna cervical en posición neutra y en flexión que mostró atrofia de la médula espinal cervical entre los niveles C5 y C7, mayor a derecha, y compresión de la médula espinal cervical secundaria a desplazamiento anterior de la pared posterior del saco tecal durante la flexión (Figura 2). En suma, dado el cuadro clínico del paciente, en conjunto con los hallazgos imagenológicos y electrofisiológicos, se realizó el diagnóstico de atrofia monomiélica distal de extremidad superior. Al paciente se encuentra actualmente en control con indicación de uso de collar cervical.

El paciente otorgó consentimiento para la publicación de su caso clínico.

\section{Discusión}

En concordancia con el caso clínico descrito, la atrofia monomiélica distal de extremidad superior corresponde a un síndrome de MNI, que afecta predominantemente los músculos de la mano y el aspecto medial del antebrazo, miotomas $\mathrm{C} 7 \mathrm{a} \mathrm{T} 1$. Clásicamente, se describe relativa preservación del trofismo del músculo braquiorradialis (miotoma C6), generando el signo clínico de "amiotrofia oblicua", elemento característico de esta enferme$\mathrm{dad}^{8}$. Adicionalmente, algunos pacientes describen exacerbación de la debilidad inducida por el frío y
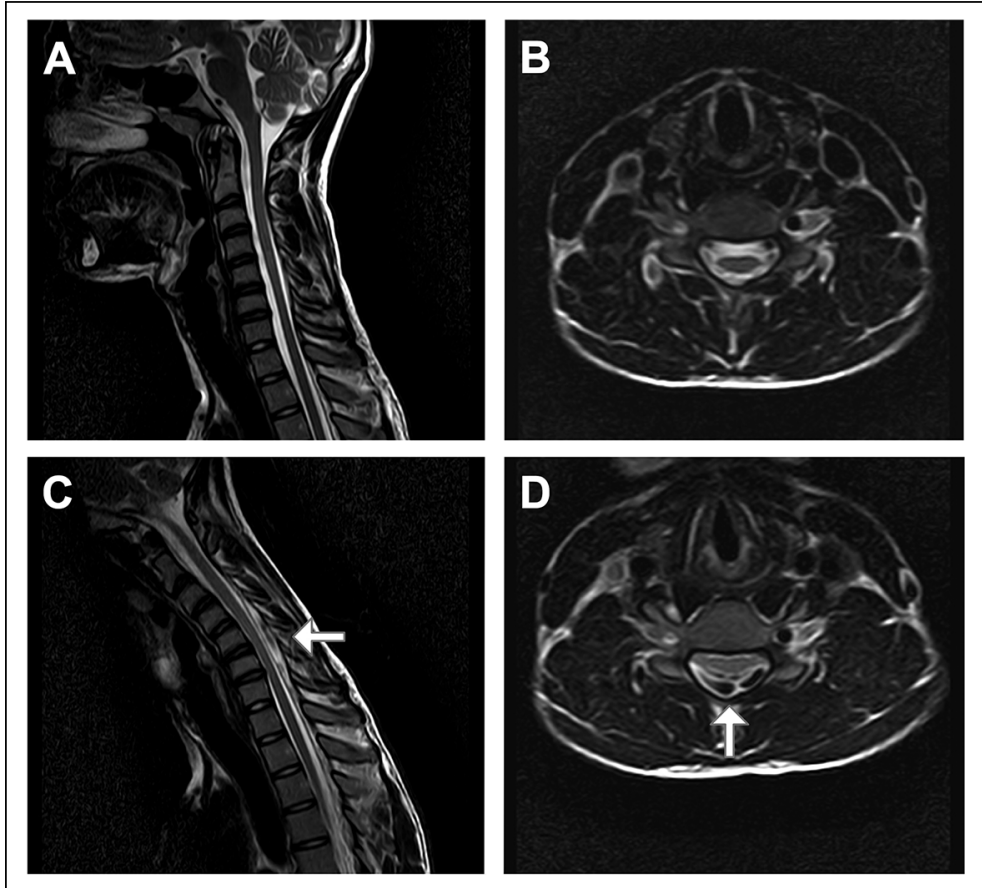

Figura 2. Resonancia magnética de columna cervical en posición neutra (A-B) y en flexión (C-D). Secuencias T2 muestran adelgazamiento de la médula espinal entre C5 a C7, mayor a derecha. En la maniobra de flexión se observa ingurgitación del plexo venoso epidural posterior, con desplazamiento del saco tecal hacia anterior, que comprime la médula espinal (flechas). 
un leve temblor durante la extensión de los dedos ${ }^{3}$, signos clínicos que nuestro paciente no presentaba. Aunque la mayoría de los pacientes tienen un compromiso focal unilateral, se ha descrito el compromiso bilateral en hasta 30\% de los casos 9 . Típicamente, los pacientes presentan indemnidad de pares craneanos, función cerebelosa, vía piramidal y función de esfínteres. Si bien la función sensitiva en la extremidad superior también está usualmente preservada, se ha descrito que $8 \%$ de los pacientes presentan leves defectos sensitivos en la extremidad comprometida, que, al igual que en nuestro paciente, presentan exámenes electrofisiológicos normales ${ }^{3}$. Aunque inicialmente la atrofia monomiélica distal de extremidad superior puede simular una ELA o una AME tipo IV, la atrofia monomiélica tiene un curso usualmente benigno, con un curso progresivo inicial, seguido de una estabilización espontánea ${ }^{10}$.

Con respecto a los exámenes complementarios, las evaluaciones electrofisiológicas e imagenológicas juegan un rol fundamental. La EMG revela pérdida de unidades motoras en los miotomas $\mathrm{C} 7$ a T1, con indemnidad de las conducciones sensitivas $^{11}$. Adicionalmente, los pacientes presentan atrofia preferente de la musculatura hipotenar (músculo ADM) por sobre la tenar (músculo APB), lo que genera un patrón de atrofia muscular llamado "síndrome inverso de la mano dividida" ("reverse split hand syndrome"). Este elemento clínico-electrofisiológico diferencia el patrón de atrofia de pacientes con ELA, en los cuales la eminencia tenar se compromete preferentemente ${ }^{12}$. Recientemente, un estudio utilizando técnicas de excitabilidad cortical mediante estimulación magnética transcraneal, determinó indemnidad de los circuitos intracorticales en pacientes con atrofia monomiélica distal de extremidad superior, lo cual permite diferenciar la enfermedad de la ELA de inicio precoz $(<45 \text { años de edad })^{13}$. En relación a los hallazgos imagenológicos, los estudios de RM cervical revelan atrofia espinal cervical (usualmente de C5 a C7), aplanamiento asimétrico espinal e hiperintesidad intramedular ${ }^{14}$. Adicionalmente, durante la flexión cervical, es posible observar un desplazamiento anterior de la pared posterior del saco tecal (duramadre dorsal) e ingurgitación del plexo venoso epidural ${ }^{15}$. Tal como lo ejemplifica nuestro paciente, es indispensable realizar RM de columna cervical en posición neutra y en flexión para realizar el diagnóstico correcto, junto con un seguimiento imagenológico si la primera RM cervical resulta normal. En relación a estos hallazgos, se ha propuesto que el proceso de mielopatía compresiva cervical podría explicarse por una potencial disparidad entre el crecimiento de la columna vertebral y el de la médula espinal ${ }^{16}$. Sin embargo, esto no explicaría la presencia de enfermedad en pacientes con RM cervical normal. Si bien los hallazgos imagenológicos previamente descritos apoyarían la hipótesis de un daño hipóxico-isquémico secundario a un fenómeno de compresión dinámico cervical, otras hipótesis etiopatogénicas incluyen potenciales procesos neurodegenerativos, autoinmunes y factores genéticos ${ }^{17,18}$. Estos últimos permitirían explicar la alta frecuencia en población asiática, junto con la existencia de casos familiares.

Con respecto a las opciones terapéuticas, se han reportado dos principales alternativas: (i) tratamiento conservador, mediante el uso de collar cervical, el cual ha reportado enlentecer la progresión clínica de la enfermedad ${ }^{19}$ y (ii) cirugía de descompresión y fijación cervical (con o sin duroplastía), la cual estaría reservada para pacientes con deterioro funcional progresivo a pesar de las medidas conservadoras ${ }^{20}$. Cabe destacar que, dado lo infrecuente de la enfermedad, no existen estudios randomizados que hayan comparado el tratamiento conservador versus el quirúrgico.

En conclusión, hemos presentado un caso clínico de atrofia monomiélica distal de extremidad superior en un paciente chileno. Dado lo infrecuente de la patología, es indispensable tener un alto grado de sospecha de la enfermedad para así realizar un diagnóstico y tratamiento en etapas tempranas. Es además relevante distinguir esta entidad de otros síndromes de MNI, como la ELA y AME, que presentan un manejo y pronóstico muy distintos.

\section{Referencias}

1. Garg N, Park SB, Vucic S, Yiannikas C, Spies J, Howells $\mathrm{J}$, et al. Differentiating lower motor neuron syndromes. J Neurol Neurosurg Psychiatry. 2017; 88 (6): 474-83.

2. Hirayama K, Tomonaga M, Kitano K, Yamada T, Kojima S, Arai K. Focal cervical poliopathy causing juvenile muscular atrophy of distal upper extremity: a pathological study. J Neurol Neurosurg Psychiatry. 1987; 50 (3): 285-90. 
3. Nalini A, Gourie-Devi M, Thennarasu K, Ramalingaiah AH. Monomelic amyotrophy: Clinical profile and natural history of 279 cases seen over 35 years (1976-2010). Amyotroph Lateral Scler Front Degener. 2014; 15 (5-6): 457-65.

4. Restuccia D, Rubino M, Valeriani M, Mirabella M, Sabatelli M, Tonali P. Cervical cord dysfunction during neck flexion in Hirayama's disease. Neurology. 2003; 60 (12): 1980-3.

5. Biondi A, Dormont D, Weitzner I, Bouche P, Chaine $\mathrm{P}$, Bories J. MR Imaging of the cervical cord in juvenile amyotrophy of distal upper extremity. AJNR Am J Neuroradiol. 1989; 10 (2): 263-8.

6. Shahrizaila N, Sobue G, Kuwabara S, Kim SH, Birks C, Fan DS, et al. Amyotrophic lateral sclerosis and motor neuron syndromes in Asia. J Neurol Neurosurg Psychiatry. 2016; 87 (8): 821-30.

7. Nascimento OJ, Freitas MR. Non-progressive juvenile spinal muscular atrophy of the distal upper limb (Hirayama's disease): a clinical variant of the benign monomelic amyotrophy. Arq Neuropsiquiatr. 2000; 58 (3B): 814-9.

8. Hassan KM, Sahni H. Nosology of Juvenile Muscular Atrophy of Distal Upper Extremity: From Monomelic Amyotrophy to Hirayama Disease-Indian Perspective. Biomed Res Int. 2013; 2013: 1-12.

9. Kao KP, Wu ZA, Chern CM. Juvenile lower cervical spinal muscular atrophy in Taiwan: report of 27 Chinese cases. Neuroepidemiology. 1993; 12 (6): 331-5.

10. Gourie-Devi M, Nalini A. Long-term follow-up of 44 patients with brachial monomelic amyotrophy. Acta Neurol Scand. 2003; 107 (3): 215-20.

11. Lyu R-K, Huang Y-C, Wu Y-R, Kuo H-C, Ro L-S, Chen $\mathrm{C}-\mathrm{M}$, et al. Electrophysiological features of hirayama disease. Muscle Nerve. 2011; 44 (2): 185-90.

12. Singh R-J, Preethish-Kumar V, Polavarapu K, Vengalil
S, Prasad C, Nalini A. Reverse split hand syndrome: Dissociated intrinsic hand muscle atrophy pattern in Hirayama disease/brachial monomelic amyotrophy. Amyotroph Lateral Scler Front Degener. 2016; 1-7.

13. Matamala JM, Geevasinga N, Huynh W, Dharmadasa T, Howells J, Simon NG, et al. Cortical function and corticomotoneuronal adaptation in monomelic amyotrophy. Clin Neurophysiol. 2017; 128 (8): 1488-95.

14. Hirayama K, Tokumaru Y. Cervical dural sac and spinal cord in juvenile muscular atrophy of distal upper extremity. Neurology. 2000; 54 (10): 1922-6.

15. Lehman VT, Luetmer PH, Sorenson EJ, Carter RE, Gupta V, Fletcher GP, et al. Cervical spine MR imaging findings of patients with Hirayama disease in North America: a multisite study. AJNR Am J Neuroradiol. 2013; 34 (2): 451-6.

16. Pradhan S, Gupta RK. Magnetic resonance imaging in juvenile asymmetric segmental spinal muscular atrophy. J Neurol Sci. 1997; 146 (2): 133-8.

17. Ito S, Kuwabara S, Fukutake T, Tokumaru Y, Hattori T. HyperIgEaemia in patients with juvenile muscular atrophy of the distal upper extremity (Hirayama disease). J Neurol Neurosurg Psychiatry. 2005; 76 (1): 132-4.

18. Lim Y-M, Koh I, Park Y-M, Kim J-J, Kim D-S, Kim $\mathrm{H}-\mathrm{J}$, et al. Exome sequencing identifies KIAA1377 and C5orf42 as susceptibility genes for monomelic amyotrophy. Neuromuscul Disord. 2012; 22 (5): 394-400.

19. Tokumaru Y, Hirayama K. [Cervical collar therapy for juvenile muscular atrophy of distal upper extremity (Hirayama disease): results from 38 cases]. Rinsho Shinkeigaku. 2001; 41 (4-5): 173-8.

20. Lu F, Wang H, Jiang J, Chen W, Ma X, Ma X, et al. Efficacy of anterior cervical decompression and fusion procedures for monomelic amyotrophy treatment: a prospective randomized controlled trial. J Neurosurg Spine. 2013; 19 (4): 412-9. 